\title{
A REVIEW OF ACOUSTIC CONSIDERATION IN PUBLIC AND MULTIFUNCTIONAL BUILDING DESIGN
}

\author{
${ }^{1}$ Oniku, S. A., and ${ }^{2}$ Bello, T. A. \\ 'Department of Physics, Federal University of Technology, Yola. \\ ${ }^{2}$ Department of Architecture, Federal University of Technology, Yola.
}

\begin{abstract}
This review paper has considered acoustics in public and multi-functional buildings design in the light of published information with the aim of bringing to our understanding the importance of acoustics liveness in multifunctional buildings. It has been shown that acoustics in buildings depend mainly on the type and use of the buildings, therefore acoustic criteria and design parameters in public and multifunctional buildings should be such that it takes into consideration the room reverberation time, background noise and sound isolation to enhance speech intelligibility and privacy. Depending on the use of the building, the acoustic materials that should be used in the design of architectural surfaces should either reflect sound, absorb sound, or diffuse sound. Therefore, for optimal intelligibility of music and speech, it must be ensured that wall and ceiling surfaces in the stage or sending end of the room are soundreflective and somewhat diffusive, to both project sounds out to an audience and to enhance performers' abilities to hear themselves and blend with one another.
\end{abstract}

Keywords: Acoustics, Public buildings, Reverberation time, Background noise, Speech intelligibility.

\section{Introduction}

Sound is a vibration in an elastic medium such as air, water, and most building materials, and the earth. Sound in air consists of a series of compressions and rarefactions due to air particles set into motion by a vibrating source. An elastic medium returns to its normal state after a force causing the vibration is removed. Sound energy progresses rapidly, producing extremely small changes in atmospheric pressure, and can travel great distances. Acoustics is therefore defined as the scientific study of sound; it is characterized by its reliance on combination of physical principles drawn from other sources (Hunt, 1978).

Sound travels at a velocity that depends primarily on the elasticity and density of the medium. In air, at normal temperature and atmospheric pressure, the velocity of sound is approximately $330 \mathrm{~m} / \mathrm{s}$. In building air distribution systems, the air velocity at registers, diffusers, and in ducts is so much slower than the velocity of sound that its effect can be neglected (Egan, 1988). For example, an extremely high air velocity of about $10 \mathrm{~m} / \mathrm{s}$ in a duct is less than 3 percent of the velocity of sound in air. However, sound may travel at a very fast velocity of $3,900 \mathrm{~m} / \mathrm{s}$ along steel pipes and duct walls. It is therefore important to block or isolate paths where sound energy can travel through building materials (called structure-borne sound) to sensitive areas great distances away where it may be regenerated as airborne sound. In buildings, the effect of temperature on sound also is negligible. For example, a $20^{\circ} \mathrm{F}$ rise or drop in room air temperature is significant in temperature range, but would cause only a 2 percent change in the velocity of sound in air (Egan, 1988). Sound reflections create standing waves that produce natural resonances that can be heard as a pleasant sensation or an annoying one (Glen, 1981). In order to obtain a good audio quality in building design it is good to use the Oscar Bonello criteria of modal density (Oscar, 1981).

In this review paper, we bring up various information available in publications to our understanding of the acoustic considerations in public and multi-functional building design. Our emphasis will be on reverberation time. Brief application of acoustic concept to specific types of spaces, including performance halls, worship buildings, offices and lecture halls are considered. 


\section{Architectural acoustics}

Architectural acoustics deals with the science of sound in buildings. McMullan (1992), stated that acoustics planning concept for buildings include, locating noisy activities away from activities that require relative quietness and noise sensitive activities away from major sources of noise. Every building type has its own acoustical requirement; therefore, it is proper to know the use of a building for good acoustic considerations. In a multi-functional public building with a wide range of activities and different acoustic requirement, care must taken in the choice of materials to ensure a balance between reverberation, reflection, absorption and isolation of sound (Karabiber, 1999). Therefore, in multi-functional public buildings (e.g. Churches and Mosques) optimal acoustic environments are required and this may be expressed in terms of some basic aural requirements such as sound audibility and speech audibility. However, short reverberation time in stressing speech intelligibility and long reverberation time in spaces where music is played is fundamental in these types of buildings (Khaiyat, 1996; Antonio, 1992).

\section{Acoustical Properties of Enclosed Space}

Basic factors that affect acoustical propagation in an enclosed space include the size and shape of the enclosure as well as the materials used in the construction of the room. However, acoustic requirements for a good acoustic in an enclosed surface (McMullan, 1992) are summarized as follows:

1. Adequate level of sound

2. Even distribution of sound to all listeners in the room

3. Rate of decay (reverberation) suitable for the type of room

4. Background noise and external noise reduced to acceptable level

5. Absence of echoes and similar acoustics defects.

Also affecting the acoustics is the size of the room, rooms for speech require a shorter reverberation time than for music. Variation in the number of people attending each programme also affects greatly the total sound absorption.

\section{Reverberation time}

Reverberation is the collection of reflected sounds from the surfaces in an enclosure like an auditorium. It is a desirable property of auditoriums to the extent that it helps to overcome the inverse square law drop off of sound intensity in the enclosure. However, if it is excessive, it makes the sounds run together with loss of articulation - the sound becomes muddy, garbled. To quantitatively characterize the reverberation, the parameter called the reverberation time is used. Reverberant sound is the collection of all the reflected sounds in an auditorium. The reverberant sound in an auditorium dies away with time as the sound energy is absorbed by multiple interactions with the surfaces of the room (Fig.1). In a more reflective room, it will take longer

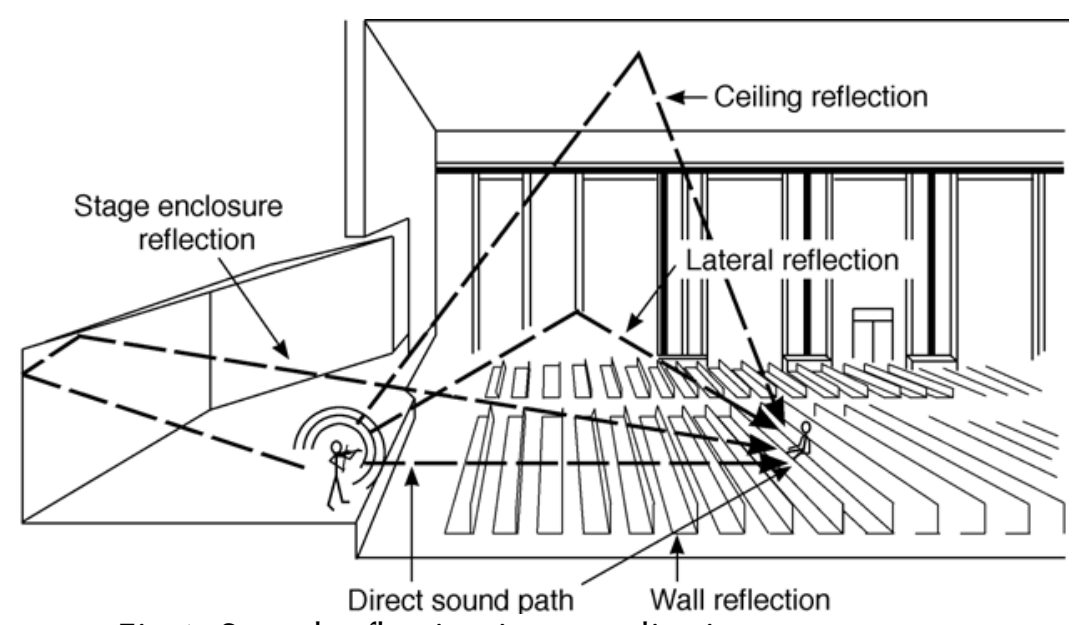

Fig.1: Sound reflection in an auditorium 
time for the sound to die away and the room is said to be 'live'. In a very absorbent room, the sound will die away quickly and the room will be described as acoustically 'dead'. But the time for reverberation to completely die away will depend upon how loud the sound was to begin with, and will also depend upon the acuity of the hearing of the observer. In order to provide a reproducible parameter, a standard reverberation time has been defined as the time for the sound to die away to a level 60 decibels below its original level.

\section{$\underset{\text { Time }}{\text { Reverberation }}=\mathrm{RT}_{60}=$ time to drop $60 \mathrm{~dB}$ below original level}

The reverberation time can be modeled to permit an approximate calculation. Sabin (1890) quoted from (www.wikipedia/reverbration time.htm) found out that the reverberation time is proportional to the dimensions of room and inversely proportional to the amount of absorption surfaces present.

Sabine's reverberation equation was developed in the late 1890s in an empirical fashion (www.wikipedia/reverbration time.htm). He established a relationship between the $R T_{60}$ of a room, its volume, and its total absorption (in sabins). This is given by the equation:

$R T_{60}=\frac{c \cdot V}{S a}$.

where $c$ is a mathematical constant measuring $0.161, V$ is the volume of the room in $\mathrm{m}^{3}, S$ total surface area of room in $\mathrm{m}^{2}$, $\mathrm{a}$ is the average absorption coefficient of room surfaces, and $\mathrm{Sa}$ is the total absorption in sabins.

The total absorption in sabins (and hence reverberation time) generally changes depending on frequency (which is defined by the acoustic properties of the space). The equation does not take into account room shape or losses from the sound travelling through the air (important in larger spaces). Most rooms absorb less in the lower frequencies, causing a longer decay time.

The reverberation time $R T_{60}$ and the volume $V$ of the room have great influence on the critical distance $d_{c}$ (conditional equation):

$d_{c}=0.057 \cdot \sqrt{\frac{V}{R T_{60}}}$

where critical distance $d_{c}$ is measured in metres, volume $V$ is measured in $\mathrm{m}^{3}$, and reverberation time $R T_{60}$ is measured in seconds.

The optimum reverberation time for an auditorium or room of course depends upon its intended use. A reverberation time of about 2.0 seconds is desirable for a medium-sized, general purpose auditorium that is to be used for both speech and music. A classroom should be much shorter, less than a second, while a recording studio should minimize reverberation time in most cases for clarity of recording (Valente et al., 2008)

When a sound wave in a room strikes a surface as shown in Fig. 1, a certain fraction of it is absorbed, and a certain amount is transmitted into the surface. Both of these amounts are lost from the room, and the fractional loss is characterized by an absorption coefficient " $a$ " which can take values between 0 and 1,1 being a perfect absorber. The effective absorbing area is a factor in determining the reverberation time of an auditorium. A table of absorption coefficients that can be used in the calculations of reverberation time with the Sabine formula is given in Table 1. 
Table 1: Absorption Coefficients (After Roth, 2007)

\begin{tabular}{|c|c|c|c|c|c|c|}
\hline \multirow{2}{*}{ Nature of Surface } & & \multicolumn{5}{|c|}{ Sound Absorption Coefficients at frequency } \\
\hline & 125 & 250 & 500 & 1000 & 2000 & 4000 \\
\hline & & & & & & \\
\hline Acoustic tile, rigid mount & 0.2 & 0.4 & 0.7 & 0.8 & 0.6 & 0.4 \\
\hline Acoustic tile, suspended & 0.5 & 0.7 & 0.6 & 0.7 & 0.7 & 0.5 \\
\hline Acoustical plaster & 0.1 & 0.2 & 0.5 & 0.6 & 0.7 & 0.7 \\
\hline ordinary plaster, on lath & 0.2 & 0.15 & 0.1 & 0.05 & 0.04 & 0.05 \\
\hline Gypsum wall board, $1 / 2$ on studs & 0.3 & 0.1 & 0.05 & 0.04 & 0.07 & 0.1 \\
\hline Plywood sheet, $1 / 4$ " on studs & 0.6 & 0.3 & 0.1 & 0.1 & 0.1 & 0.1 \\
\hline Concrete block unpainted & 0.4 & 0.4 & 0.3 & 0.3 & 0.4 & 0.3 \\
\hline Concrete block painted & 0.1 & 0.05 & 0.06 & 0.07 & 0.1 & 0.1 \\
\hline Concrete, poured & 0.01 & 0.01 & 0.02 & 0.02 & 0.02 & 0.03 \\
\hline Brick & 0.03 & 0.03 & 0.03 & 0.04 & 0.05 & 0.07 \\
\hline Vingl tile on concrete & 0.02 & 0.03 & 0.03 & 0.03 & 0.03 & 0.02 \\
\hline Heavy carpet on concrete & 0.02 & 0.06 & 0.15 & 0.4 & 0.6 & 0.6 \\
\hline Heavy carpet on felt backing & 0.1 & 0.3 & 0.4 & 0.5 & 0.6 & 0.7 \\
\hline Platform floor, wooden & 0.4 & 0.3 & 0.2 & 0.2 & 0.15 & 0.1 \\
\hline Ordinary window glass & 0.3 & 0.2 & 0.2 & 0.1 & 0.07 & 0.04 \\
\hline Heavy plate glass & 0.2 & 0.06 & 0.04 & 0.03 & 0.02 & 0.02 \\
\hline Draperies, medium velour & 0.07 & 0.3 & 0.5 & 0.7 & 0.7 & 0.6 \\
\hline Upholstered seating, unoccupied & 0.2 & 0.4 & 0.6 & 0.7 & 0.6 & 0.6 \\
\hline Upholstered seating, occupied & 0.4 & 0.6 & 0.8 & 0.9 & 0.9 & 0.9 \\
\hline Wood seating, unoccupied & 0.02 & 0.03 & 0.03 & 0.06 & 0.06 & 0.05 \\
\hline Wooden pew's, occupied & 0.4 & 0.4 & 0.7 & 0.7 & 0.8 & 0.7 \\
\hline
\end{tabular}

\section{Design Considerations}

Acoustics in buildings depend mainly on the type and use of the buildings. Public and multi-functional government buildings by nature are used for public assembly, concert halls, schools, hospitals and hotels. Acoustic criteria and design parameters in such buildings should be such that it takes into consideration the room reverberation time, background noise and sound isolation to enhance speech intelligibility and privacy. Depending on the use of the building, the acoustic materials that should be used in the design of architectural surfaces should either, reflect sound, absorb sound, or diffuse sound.

The sound wave has reflections at the walls, floor and ceiling of the room as shown in (Fig.1). The incident wave then has interference with the reflected one. This action creates standing waves that generate nodes and high pressure zones (Leo, 1954). The mode spacing is a very important factor especially in small and medium size rooms like recording studios, home theatres, broadcasting studios and concert halls. Some methods (www.wikipedia/room acoustics.htm) were created from 1940 - 1981 to obtain the best mode spacing to avoid sound colouration, but none of them succeed, because the mode spacing is not only a geometric problem. In order to solve this problem, Oscar, (1981) introduced the Modal Density concept that solves the problem using the concepts from Psychoacoustics. This new Bonello's Criterion as it was named, analyzes the first 48 room modes and plots the number of modes in each one-third of octave. The curve must increase monotonically (each one-third of octave must have more modes than the preceding one). This Criterion is now the standard method of designing the room dimensions (Glen, 1981).

After determining the best dimensions of the room, using Modes Density criteria, the next step is finding the correct reverberation time (www.wikipedia/reverberation time.htm). The reverberation time depends on the use of the room. Times of about 1.5 to 2 seconds are needed for Opera Theaters and Concert Halls. For Broadcasting and Recording studios and Conference rooms and lecture theatre, values under one second are frequently used. The recommended 
Reverberation Time is always a function of the volume of the room. Several authors give their recommendations ((Egan, 1988; Leo, 1954). A good approximation for Broadcasting Studios and Conference Rooms is:

$$
\operatorname{TR}[1 \mathrm{khz}]=[0.4 \log (\mathrm{V}+62)]-0.38
$$

$T R$ in seconds and $V=$ volume of the room in $\mathrm{m}^{3}$ (Oscar, 1979).

The ideal RT must have the same value at all frequencies from 30 to $12,000 \mathrm{~Hz}$ or at least, is acceptable to have a linear rising from $100 \%$ at $500 \mathrm{~Hz}$ to $150 \%$ down to $62 \mathrm{~Hz}$

In order to get the calculated RT in a room, several acoustics materials as described in several books (Michael, 1977; Egan, 1988; Egan et al., 1999 and David and Chair, 2003) are considered. A valuable simplification of the task was also proposed by Oscar (1979).

For optimal intelligibility of music and speech, it must be ensured that wall and ceiling surfaces in the stage or "sending" end of the room are sound-reflective and somewhat diffusive, to both project sounds out to an audience and to enhance performers' abilities to hear themselves and blend with one another. In this case the room shape is very important for providing the necessary side wall reflections that contribute to an accurate sense of spaciousness and fullness of sound in the space. Rooms based on the rectangular form (with added wall shaping) often provide the strongest coverage of side wall, or lateral, reflections. Wide fan shapes and semicircular floor plans focus sound very unevenly causing "hot spots" and "dead zones" of sound (Egan et al., 1999).

\section{Conclusion}

This paper has reviewed mainly the works of Egan, 1988; Egan et al., 1999 and a few others and has concluded that reverberation time, which is dependent on the volume and the total absorption of the room is the most important acoustic parameter in building designs. Basic factors that affect a room's reverberation time include the size and shape of the enclosure as well as the materials used in the construction of the room. Every object placed within the enclosure can also affect this reverberation time, including people and their belongings. Therefore, depending on the type and use of the building, the acoustic materials to be used should provide for optimal intelligibility of music and speech. In a room that is meant for learning and teaching, a good aural communication between the teacher and the students are of utmost important, therefore when selecting finishes, a proper balance between sound-absorptive and sound-reflective materials is necessary to produce an environment that is not overly reverberant.

\section{References}

Antonio, P. D. (1992). Acoustical control of worship places. Journal of JBD sound acoustics lab; $1-3$

Benade, A. (1976). Fundamentals of Musical Acoustics, New York, NY, United States: Dover.

David, E. M. and Chair, (2003). Architectural acoustics and noise: Acoustic design of Government buildings. $146^{\text {th }}$ meeting of the Acoustic Society of America, Austin, Texas.1014 Nov., 2003.

Egan, M. D. (1988). Architectural Acoustics. New York: McGraw-Hill.

Egan, M. D., Steven, H. and Christopher, J. (1999). Acoustics: Theory and Applications, Chapter 8, 102-116, New York: McGraw-Hill.

Glen Ballou. (1981). Handbook for Sound Engineers. Howards Sams Editors, page 56.

Hunt, T. F. V. (1978). Acoustic phenomenon: Origin in acoustics, Yale University press, page 154

Karabiber, Z. (1999). Acoustics problems in mosques, a case study of three mosques, Istanbul. Journal of acoustical society of America. Vol 105, issue 2.

Khaiyat, S. A. (1996). Mosque acoustics. Journal of acoustical society of America. $132^{\text {nd }}$ meeting laid language papers, 1-4.

Leo Beranek. (1954). Acoustics. chapter 10, McGraw Hill Books. 
McMullan, R. (1992). Room acoustics: Environmental science in buildings, $3^{\text {rd }}$ edition, London. The McMullan press limited.

Michael, R. (1977). Acoustic Design and Noise Control. Chemical Publishing, NY.

Oscar Bonello, (1979). A new computed aided method for the complete design of broadcasting and recording studios. IEEE International conference on Acoustics, Speech \& Signal Procession, Washington

Oscar Bonello, (1981). A new criterion for the distribution of normal room modes. Journal of the Audio Engineering Society (USA) Vol. 29, No. 9

Roth, L. M. (2007). Understanding Architecture. Westview Press. pp. 104-105.

Valente, M., Holly H.D. and Ross J. R. (2008). Audiology. Thieme. pp. 425-426. www.wikipedia/Archtectural acoustics.htm www.wikipedia/reverbrationtime.htm www.wikipedia/roomacoustics.htm 